\title{
Effective Valencies of Liquid 3d Transition Metals Estimated from the Gibbs-Bogoliubov Variational Method Using the One-Component Plasma Reference System
}

\author{
T. Arai, S. Naito and I. Yokoyama \\ Department of Mathematics and Physics, National Defense Academy, \\ Yokosuka 239, Japan
}

(Received December 21, 1992; final form February 10, 1993)

\begin{abstract}
We present the effective valencies of liquid $3 \mathrm{~d}$ transition metals estimated from the Gibbs-Bogoliubov variational method using the classical one-component plasma reference system. The effective pair interactions are calculated within the framework of the simple-metal theory. We employ the Ashcroft empty-core form for the s-electron pseudopotential. The value of the core radius is uniquely determined by the density of the system of interest. The effective valencies determined from the variational method are compatible with those obtained from other theoretical works. The calculated values of the long-wavelength limit of the structure factor are in excellent agreement with the available experimental values.
\end{abstract}

\section{INTRODUCTION}

Recently Hausleitner and Hafner /1/ and Hausleitner et al. $/ 2 /$ have presented quite successful ab initio calculations of the structures and thermodynamic properties of liquid transition metals based on effective pair interactions calculated within a hybridized nearly-free electron tight binding theory and on a thermodynamic variational method with a hard-sphere Yukawa reference system. These authors obtained good values for the liquid metal entropies and calculated the pair distribution functions and the static structure factors, which are in reasonable agreement with the available diffraction data. These authors also found that the characteristic feature of the interionic interactions in transition metals lies in the extreme softness of the repulsive part and that this is readily understood in terms of competing $s$ and delectron interactions. Quite recently Bretonnet et al. /3/ reported the structural results of the Gibbs-Bogoliubov (GB) variational scheme with a charged-hard sphere (CHS) reference system. These have been studied using effective potentials /4/ that combine the empty-core model used to describe the nearly-free electron band and a d-band contribution deduced via an inverse scattering approach. Their effective pair potentials are shallower and shifted towards the larger values of $r$ than the corresponding WillsHarrison potentials used by other authors $/ 1,5 /$. The calculated results for the structure factor agree reasonably well with the available experimental data. The CHS system includes the size effect of the positive ions interacting via a Coulomb potential in a uniform background of negative charge, but this system is essentially the 
same as the one-component plasma (OCP) model because the optimized values found for the hard-sphere diameters reveal that the ions never sample the hard cores. Their work obviously suggests using a soft-core reference system such as the $\mathrm{OCP}$ for liquid transition metals.

The OCP system /6-8/ is characterized by an ionsphere radius $a=(3 / 4 \pi n)^{1 / 3}$, with $n$ being the number density of ions, and a plasma parameter, $\Gamma=(\mathrm{Ze})^{2} / a \mathrm{k}_{\mathrm{B}} T$. For liquid $3 \mathrm{~d}$ transition metals, this OCP approach was first investigated by Itami and Shimoji /9/ and then by Itoh et al. /10\%. Its usefulness for the thermodynamic properties of the transition metals was recently reported by Yokoyama and Naito /11,12/. The calculated values of the entropy, the specific heats at constant pressure and volume, the ratio of the specific heats, the isothermal compressibility and the structure factor (except for liquid Sc, Ti and V) are in reasonable agreement with the available observed data. All the calculations /10-12/ mentioned above, however, were performed using the empirical value of the plasma parameter, $\Gamma=110$, the physical meaning of which is not yet clear from the fundamental point of view of liquid metal theory.

The purpose of the present paper is twofold: first, to investigate the validity of the empirical value of $\Gamma=110$ in terms of the GB variational method using the OCP reference system; second, to calculate the longwavelength limit of the structure factor using the values of $\Gamma$ and the effective valencies obtained from the GB method. The paper is organized in the following way: In Section 2, we present a brief description of the GB formalism necessary to carry out a variational calculation; in Section 3, we refer to the pseudopotential and the screening function used in the present work; numerical results are presented and discussed in Section 4; and in Section 5, conclusions drawn from the present work are presented.

\section{THE THERMODYNAMIC VARIATIONAL METHOD}

We assumed that as a first approximation to the electronic structure of a transition metal with $Z$ valence electrons per ion, $Z_{d}$ electrons are incorporated in the non-overlapping ion core, the remaining $Z_{s}=Z-Z_{d}$ electrons per ion are in the free-electron-like states, and their coupling is weak. This idea of separation between core and conduction electron states corresponds to the proposal by Wills and Harrison /13/, although the bonding contribution and the repulsive energy from the d-electrons are not explicitly taken into account here. Thus the calculation throughout this work is based on a straightforward application of the simple-metal theory. It may not be at all clear why a theory developed for simple(s-p) metals should be meaningful for transition metals. However, according to the recent Regnaut's analysis /5/ of the liquid structure of $3 \mathrm{~d}$ transition metals from the Wills-Harrison (WH) model, the predictions of the WH potential depend critically on the cancellation between the two large repulsive and attractive terms arising respectively from the simple-metal-like contribution and the d-band contribution. Both the position and the intensity of the attractive well are strongly modified by changing the value of the Ashcroft empty-core radius and these modifications are as large as those induced by the d-band contribution itself. Regnaut's findings were confirmed by the recent work of Hausleitner et al. /2/. The latter authors concluded that improvements in the description of the structure of liquid transition metals require a shallower potential well shifted towards a larger value of $r$ than that predicted by WH. Since the structure factor data from well below the first peak to high wave number, $k$, are mainly described by the repulsive part corresponding to the energy of the order of $k_{B} T$ measured from the bottom of the effective pair potential, it is possible to adjust its gradient by using an empty-core potential with an effective charge. Bearing this in mind, we assume that the simple-metal theory can describe the structures of liquid transition metals when the pseudopotential parameters are properly selected.

We view a liquid metal as a collection of ions with potential energy described by a structure-independent term, $u_{0}(n)$, and a pairwise interaction, $u(r, n)$ : both terms depend in principle on $n$, the number density of the ions. The potential energy per ion according to Hasegawa and Watabe /14/ is then written using the Fourier transform as 


$$
\begin{aligned}
\mathrm{U}= & u_{0}(n)+\frac{1}{2 N} \sum_{\mathbf{k}} V^{-1} \\
& \sum_{\mathrm{i} \times \mathrm{j}} \exp \left[\mathbf{i k} \cdot\left(\mathbf{r}_{i}-\mathbf{r}_{j}\right)\right] u(k, n)
\end{aligned}
$$

where $N$ is the total number of ions, $V$ is the total volume of the system and

$$
u(k, n)=\int u(r, n) \exp (\mathrm{ik} \cdot \mathbf{r}) \mathrm{d} \mathbf{r} .
$$

The Helmholtz free energy of the system defined by Eq. 1 is now approximated by the use of the GB inequality $/ 15,16 /$ to

$$
F \leq F_{0}+\left\langle H-H_{0}\right\rangle
$$

where $F_{0}$ is the Helmholtz free energy (per ion) of the reference system, and $H$ and $H_{0}$ denote the Hamiltonians of the real and reference systems, respectively. Hence Eq. 3 states that the free energy of the actual system is bounded above by that of the reference system plus the difference in the potential energy between the actual and reference systems averaged over all configurations of the reference system. The approximation of employing the OCP as the reference leads to the following expression for the free energy /17/:

$$
\begin{aligned}
F= & F_{\mathrm{id}}+u_{0}(n)-T \Delta S_{0}+\frac{1}{2} n u(0, n) \\
& +\frac{1}{2} \int \frac{\mathrm{dk}}{(2 \pi)^{3}} u(k, n)\left(a_{0}(k a, \Gamma)-1\right)
\end{aligned}
$$

where $a_{0}(\mathrm{k} a, \Gamma)$ is the OCP structure factor and $\Delta S_{0}$ is the excess entropy given as

$$
\begin{aligned}
\Delta S_{0} / \mathrm{k}_{\mathrm{B}}= & -2.83632 \Gamma^{1 / 4}+0.89770 \Gamma^{-1 / 4} \\
& +0.800491 \mathrm{n} \Gamma+1.78559
\end{aligned}
$$

$F_{\text {id }}$ is the free energy of an ideal gas,

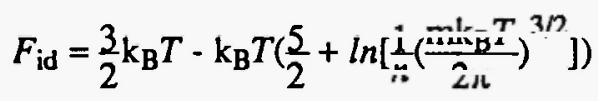

where $m$ is the mass of ion, $k_{B}$ the Boltzmann constant and $T$ the absolute temperature. The right-hand side of Eq. 4 is minimized with respect to $\Gamma$.

\section{PSEUDOPOTENTIAL AND SCREENING FUNCTION}

We now use the pseudopotential theory to describe the functions $u(k, n)$ and $u_{0}(n)$ which are necessary to the formalism above. It is convenient to write the linear response form (in atomic units) $/ 18,19 /$

$$
u(k, n)=\left(4 \pi Z_{s}^{2} / k^{2}\right)\left(1-F_{N}(k, n)\right)
$$

where $Z_{s}$ is the effective valency and

$$
\mathrm{F}_{\mathrm{N}}(k, n)=\left(k^{2} \mathrm{v}(k) / 4 \pi Z_{\mathrm{s}}\right)^{2}(1-1 / \varepsilon(k, n))
$$

is the normalized energy-wavenumber characteristic. Here $\mathrm{v}(k)$ is the local pseudopotential, and $\varepsilon(k, n)$ is the dielectric screening function which may be written as

$$
\varepsilon(k, n)=1+\left(4 \pi / k^{2}\right) \Pi(k, n)
$$

where $\Pi(k, n)$ is the electron-gas polarization function and

$$
\Pi(k, n)=\frac{\left(k_{\mathrm{F}} / \pi^{2}\right) \mathrm{f}(\mathrm{x})}{1-\left(4 \mathrm{k}_{\mathrm{F}} / \pi \mathrm{k}^{2}\right) \mathrm{G}(\mathrm{x}) \mathrm{f}(\mathrm{x})} .
$$

$k_{\mathrm{F}}$ is the Fermi wavenumber and

$$
f(x)=\frac{i}{2}+\left[\left(x^{2}-1\right) / 4 x\right] \ln \left|\frac{1-x}{i+\lambda i}\right|, x=k / 2 k_{F}
$$

and $\mathrm{G}(\mathrm{x})$ incorporates the effects of exchange and correlation in the electron gas.

In the present work, we used the Ashcroft /20/ empty-core form

$$
\mathrm{v}(k)=-\left(4 \pi Z_{s} / k^{2}\right) \cos \left(k r_{\mathrm{c}}\right)
$$

for the pseudopotential, $r_{c}$ being the core radius, and the Geldart-Vosko expression /21/

$$
G(x)=x^{2}\left[2 x^{2}+\left(1+0.153 \mu^{2}\right)^{-1}\right], \mu^{2}=1 / \pi k_{F}
$$


is used to describe the electron gas.

The volume term $u_{0}(n)$ is given by

$u_{0}(n)=F_{\mathrm{eg}}+F_{\text {self }}-\frac{\mathrm{n}}{2} \frac{\mathrm{d}^{2}}{\mathrm{dn}^{2}}\left(\mathrm{n} F_{\mathrm{eg}}\right)$

where $F_{\text {eg }}$ and $F_{\text {self }}$ are the Helmholtz free energy (per ion) of the associated uniform electron gas and the selfenergy of an ion due to the electronic screening effect, expressed as

$$
\begin{gathered}
\mathrm{F}_{\mathrm{eg}}-\left[\frac{3}{10} \mathrm{k}_{\mathrm{F}}^{2}-\frac{3 \mathrm{k}_{\mathrm{F}}}{4 \pi}-\left(0.0474+0.0155 \ln \mathrm{k}_{\mathrm{F}}\right)\right. \\
\left.-\frac{1}{12}\left(\pi \mathrm{k}_{\mathrm{B}} \mathrm{T}\right)^{2} \frac{\mathrm{N}\left(\mathrm{E}_{\mathrm{F}}\right)}{\mathrm{n}}\right] \mathrm{Z}_{\mathrm{s}} \\
\mathrm{F}_{\mathrm{self}}=-\frac{1}{2 N} \sum_{\mathrm{k} \neq 0} \frac{4 \pi \mathrm{Z}_{\mathrm{s}}^{2}}{\dot{k}^{2}} n \mathrm{~F}_{\mathrm{N}}(k, n) .
\end{gathered}
$$

$N\left(E_{F}\right)$ is the density of the states per unit volume at the Fermy energy, and we use the rectangular model $/ 22 /$ for this. However, the volume term has no direct relevance to the present work because only the structure-dependent part of the Helmholtz free energy in Eq. 4 is minimized with respect to $\Gamma$.

The $k=0$ limit has a special significance and needs to be written explicitly. For the Ashcroft empty-core form

$$
u(0, n)=4 \pi Z_{\mathrm{s}}^{2}\left[r_{\mathrm{c}}+\frac{\pi}{4 k_{\mathrm{F}}} \cdot\left(\frac{1}{4 k_{\mathrm{F}}^{2}}+\frac{0.153}{4 \pi \mathrm{k}_{\mathrm{F}}^{3}}\right)\right]
$$

for the Geldart-Vosko expression. In the present work, all the electronic quantities $k_{\mathrm{F}}, \varepsilon(k, n)$ and $\mathrm{v}(k)$ are evaluated by using the effective valency, $Z_{s}$.

\section{RESULTS AND DISCUSSION}

\subsection{Effective Valency, $Z_{s}$}

In order to complete the description of the $\mathrm{GB}$ variational approach, we take an OCP structure factor, $a_{0}(\mathrm{k} a, \Gamma)$, developed by Chaturvedi et al. /6-8/. The input data used in this work are given in Table 1. The values of $r_{c}$ are determined through the relation, $k o r_{c}=$ $\pi / 2$. Here $k_{0}$ denotes the position of the first node of the Ashcroft pseudopotential in $k$-space, and this is chosen to get the correct number of degrees of freedom, i.e., $k_{0}=\left(18 \pi^{2} n\right)^{1 / 3}$. This is based on the assumption that all or almost all the elementary excitations in liquid metals can be attributed to the density fluctuations $/ 24 /$. If we accept this assumption, the zeroth order picture is of longitudinal phonons with $\mathbf{k}$-vectors occupying an

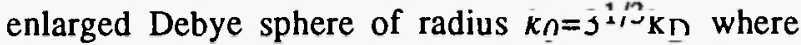
$\mathrm{k}_{\mathrm{D}}=\left(6 \pi^{2} n\right)^{1 / 3}$. Hence the value of $r_{c}$ is uniquely determined by the density of the system of interest. The values of $r_{c}$ given in Table 1 are comparable, though a little small, to those listed in Table 2 of Hausleitner et al. $/ 2 /$.

In Fig. 1, the calculated Helmholtz free energies are plotted against $\Gamma$ for the liquid Ti, V, Cr, Mn, Fe, Co and $\mathrm{Ni}$ near their melting points. Calculations were performed using trial valencies denoted in the figure caption. These trial valencies are determined from $\Gamma=110$ through the relation of $Z_{s}=\sqrt{\Gamma a k_{\mathrm{B}} T}$ where $a=$ $(3 / 4 \pi n)^{1 / 3}$. As can be seen from these figures, the position of the minimum of the free energy is found around $\Gamma=105$ for all the cases presently studied. If this were found around $\Gamma=110$, it would be very pleasing. However, the variationally-determined value of $\Gamma$ is fairly close to 110 , so the validity of the empirical value of $\Gamma=110$ used in the previous works seems to be well supported by the thermodynamic variational method. The value of the effective valency $Z_{s}$ is determined from $\Gamma=105$ through the relation of $Z_{s}=\sqrt{\Gamma a k_{\mathrm{B}}} T$, which gives the minimum of the calculated Helmholtz free energy and is summarized in Table 1. Regarding the effective valency, Hausleitner and Hafner / $/$ and Regnaut $15 /$ took $Z_{s}=1.5$, following $\mathrm{WH} / 13 /$, for all the elements of the $3 \mathrm{~d}$ series. Hausleitner et al. $/ 2 /$ took $Z_{s}$ from the self-consistent calculation of Moruzzi $/ 25 /$. On the other hand, Bretonnet et al. $/ 3 /$ used $Z_{s}=1.2$ for all the elements in the $3 \mathrm{~d}$ series. It can be seen from Table 1 that the present results for $Z_{s}$ are completely compatible with those used by these authors. Incidentally, the calculated values of the free energy for $\Gamma=120$ are distinctly high for all the cases studied. This appears to be caused by the values of the several parameters involved in the OCP structure factor. 
TABLE 1

Input data $\left(T, \rho\right.$ and $\left.r_{c}\right)$, the effective valency $Z_{s}$ and the long-wavelength limit of the structure factor $s(0)$ calculated from Eq. 18. $T$ is the temperature, $\rho$ the mass density, $r_{c}$ the empty-core radius and $\Gamma$ the plasma parameter which gives the minimum of the Helmholtz free energy. $Z_{s}=\sqrt{\Gamma a k_{\mathrm{B}} T}$ where $a=(3 / 4 \pi n)^{1 / 3}$ with $n$ the number density of ions.

\begin{tabular}{|c|c|c|c|c|c|c|c|}
\hline & \multirow{2}{*}{$\begin{array}{c}T \\
(\mathrm{~K})\end{array}$} & \multirow{2}{*}{$\begin{array}{c}\rho \\
\left(\mathrm{g} . \mathrm{cm}^{-3}\right)\end{array}$} & \multirow[t]{2}{*}{$\Gamma$} & \multirow[t]{2}{*}{$Z_{s}$} & \multirow{2}{*}{$\begin{array}{c}r_{c} \\
\text { (a.u.) }\end{array}$} & \multicolumn{2}{|c|}{$s(0)$} \\
\hline & & & & & & calc & expt.* \\
\hline $\mathrm{Ti}$ & 1973 & 4.15 & 105 & 1.43 & 1.413 & 0.024 & 0.020 \\
\hline $\mathrm{V}$ & 2173 & 5.36 & 105 & 1.46 & 1.324 & 0.022 & 0.025 \\
\hline $\mathrm{Cr}$ & 2173 & 6.27 & 105 & 1.42 & 1.264 & 0.022 & 0.021 \\
\hline $\mathrm{Mn}$ & 1533 & 5.97 & 105 & 1.22 & 1.310 & 0.022 & 0.024 \\
\hline $\mathrm{Fe}$ & 1833 & 7.01 & 105 & 1.30 & 1.249 & 0.021 & 0.020 \\
\hline Co & 1823 & 7.70 & 105 & 1.29 & 1.231 & 0.021 & 0.019 \\
\hline $\mathrm{Ni}$ & 1773 & 7.72 & 105 & 1.27 & 1.231 & 0.021 & 0.020 \\
\hline
\end{tabular}

* taken from Waseda and Ueno $/ 23 /$

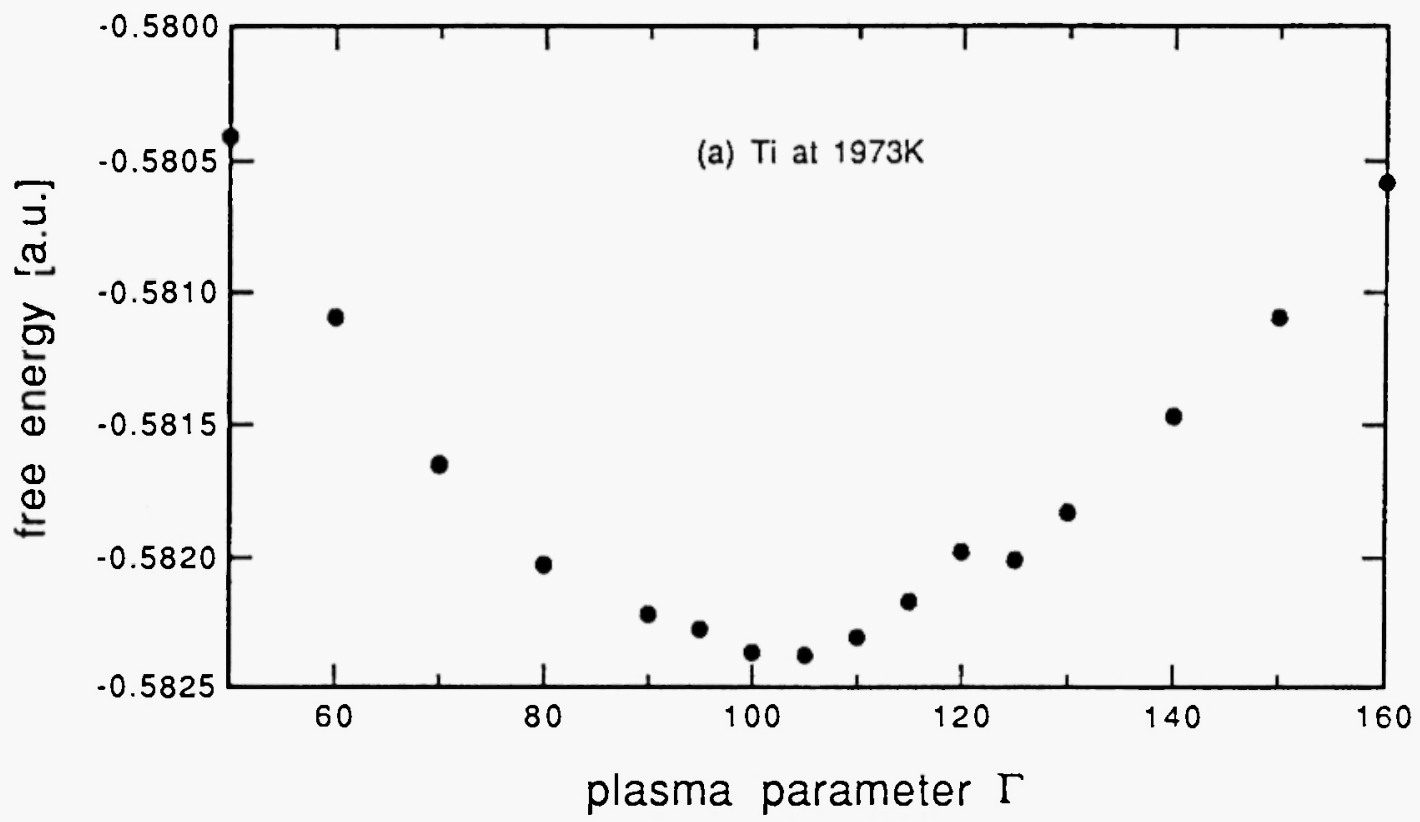

Fig. 1: Helmholtz free energy for the liquid $3 d$ transition metals versus plasma parameter $\Gamma$. We used the Ashcroft empty-core pseudopotential with the screening function of Geldart and Vosko $/ 21 /$ and the OCP structure factor due to Chaturvedi et al. /6-8/. The position of the minimum of free energy is found around $\Gamma=105$ for all the metals studied: (a) $\mathrm{Ti}$ at $1973 \mathrm{~K}$ (trial valency $\mathrm{Zs}=1.47$ ); (b) $\mathrm{V}$ at $2173 \mathrm{~K}$ (trial valency $\mathrm{Zs}=1.49$ ); (c) $\mathrm{Cr}$ at $2173 \mathrm{~K}$ (trial valency $\mathrm{Zs}=1.46$ ); (d) $\mathrm{Mn}$ at $1533 \mathrm{~K}$ (trial valency $\mathrm{Zs}=1.25$ ); (e) $\mathrm{Fe}$ at $1833 \mathrm{~K}$ (trial valency $\mathrm{Zs}=1.33$ ); (f) Co at $1823 \mathrm{~K}$ (trial valency $\mathrm{Zs}=$ 1.32); (g) Ni at 1773K (trial valency $\mathrm{Zs}=1.30$ ). 

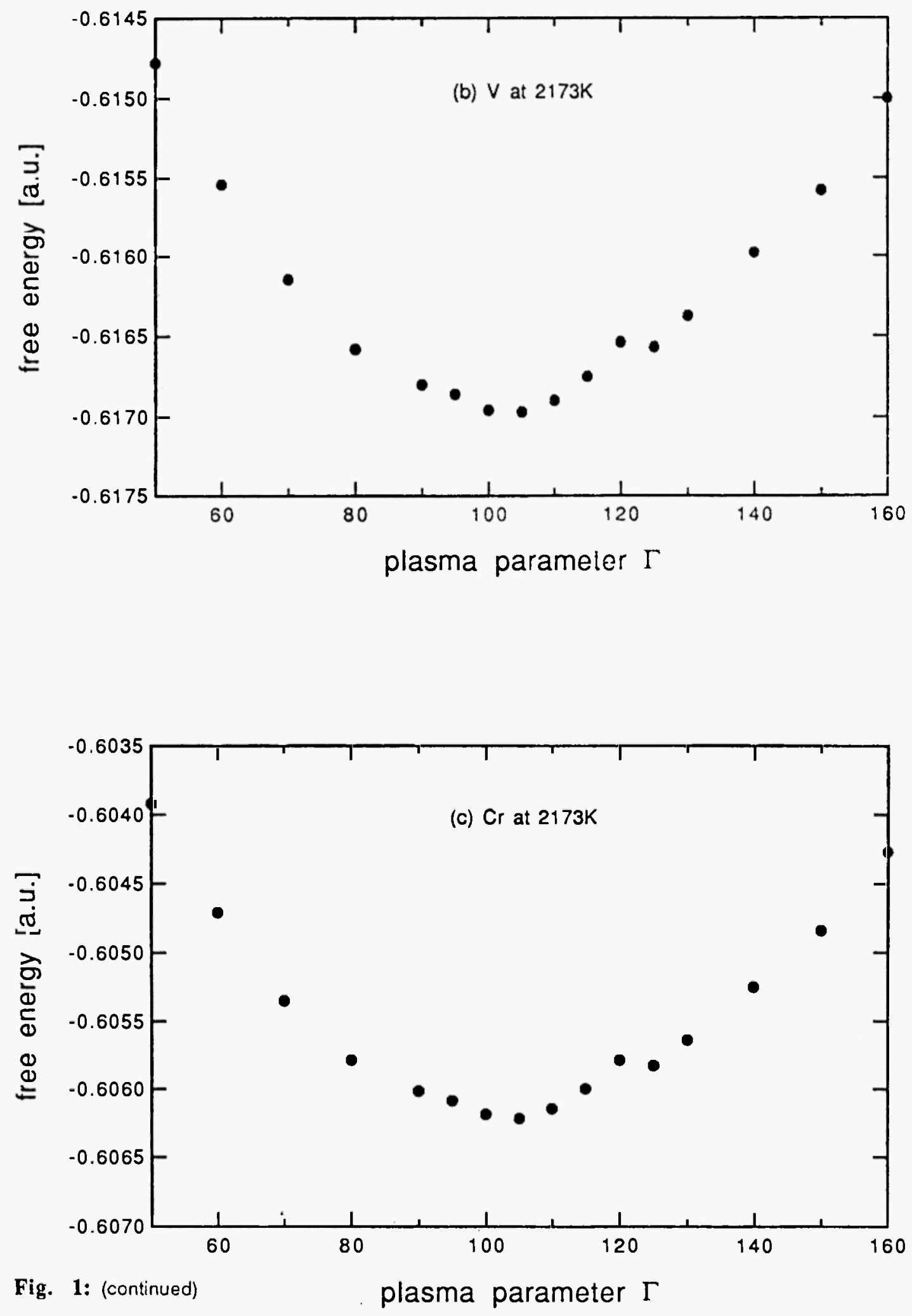

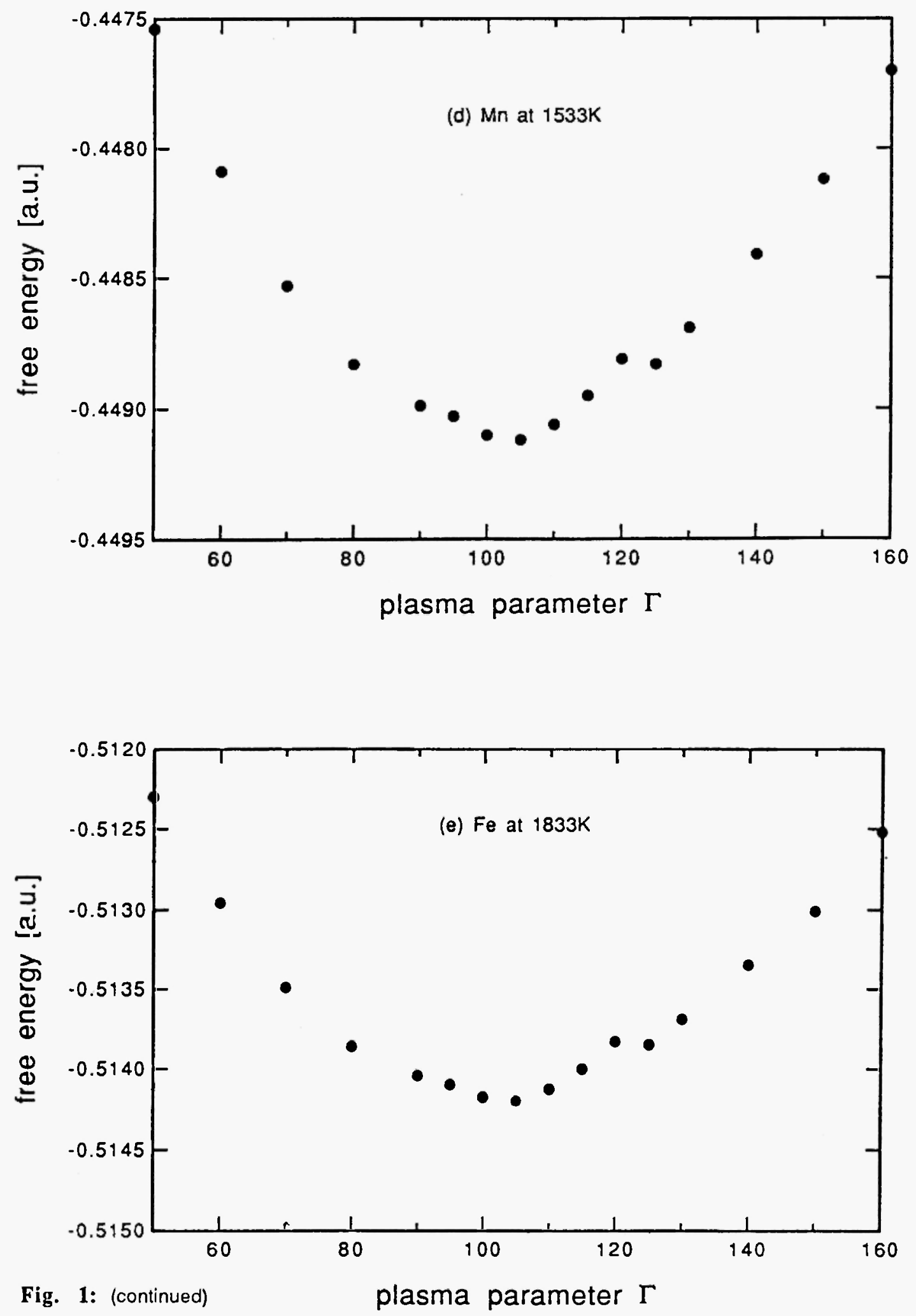

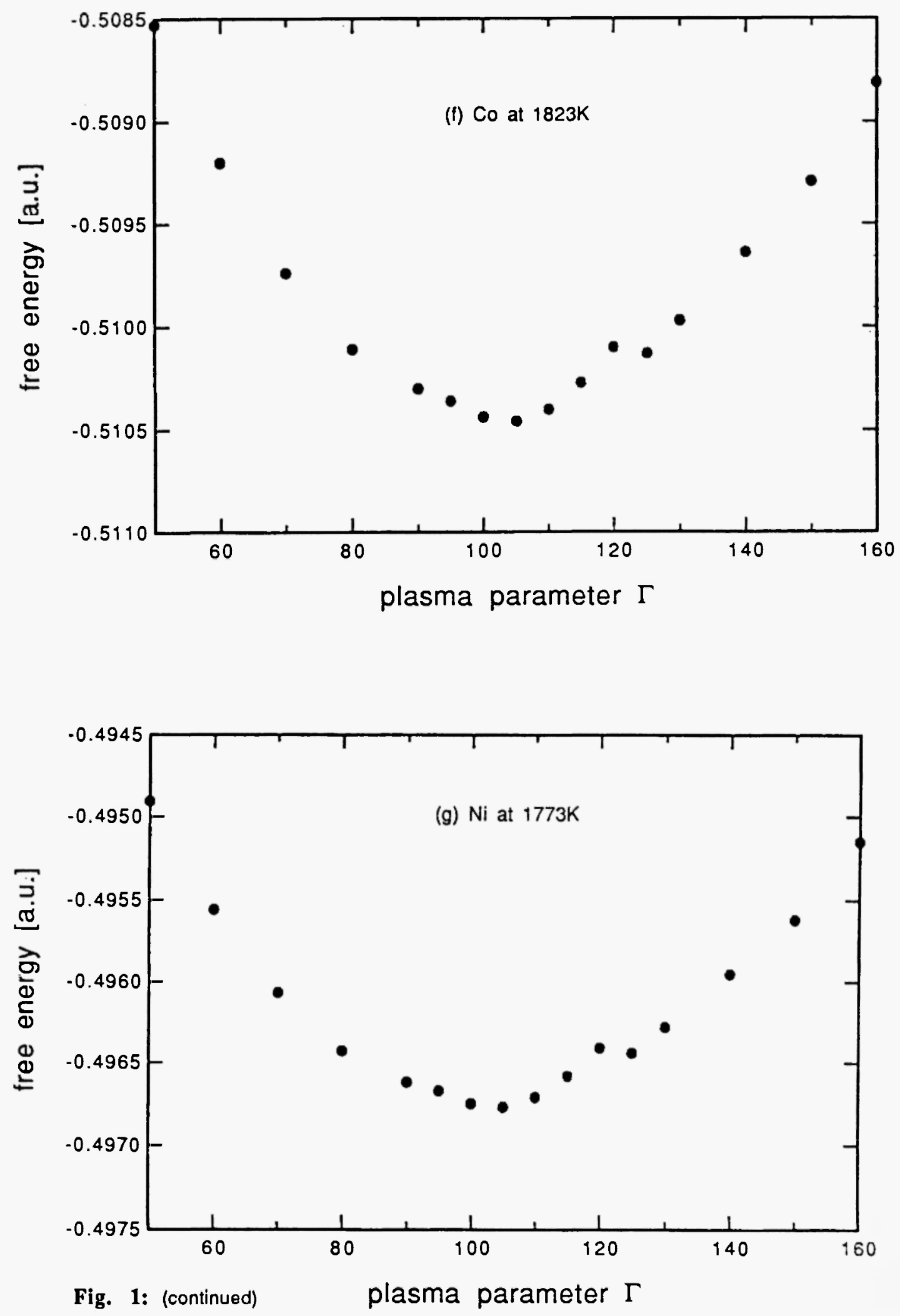


\subsection{Long-Wavelength Limit of Structure Factor, s(0)}

According to Chaturvedi et al. /8/, the longwavelength limit of the structure factor, $s(0)$, for liquid metals can be written as

$s(0)=\left(k_{\mathrm{DH}}^{2} / k_{\mathrm{e}}^{2}+k_{\mathrm{DH}}^{2} / k_{\mathrm{i}}^{2}+{ }_{k_{\mathrm{DH}}}^{2} r_{\mathrm{c}}^{2}\right)^{-1}$

where

$$
k_{\mathrm{DH}}=\left(4 \pi n Z_{\mathrm{s}}^{2} / k_{\mathrm{B}} T\right)^{1 / 2}
$$

is the Debye-Hückel inverse screening length, and $k_{\mathrm{i}}$ is the "inverse screening length" of the OCP given by

$$
\begin{aligned}
k_{\mathrm{DH} /}^{2} k_{\mathrm{i}}^{2}= & 1-0.398415 \Gamma \\
& +0.31122 \Gamma^{1 / 4}-0.1850 .
\end{aligned}
$$

The quantity $k_{\mathrm{e}}$ in Eq. 18 is the inverse screening length of the electron gas and is related to $\varepsilon(k, n)$ as

$$
\lim _{k \rightarrow U} \varepsilon(k, n)=1+k_{e}^{2} / k^{2}
$$

where

$$
{ }_{k}^{2}=\frac{4 \pi k \mathrm{~F}^{3}}{(\pi k \mathrm{~F})^{2}-\pi k \mathrm{~F}-0.153}
$$

for the Geldart-Vosko expression. In Table 1, the calculated values of $s(0)$ are summarized together with the experimental data $/ 23 /$. It can be seen from the table that agreement between the calculated and the experimental $s(0)$ is quite good, and, therefore, good values for the isothermal compressibilities are assured using these $Z_{s}, \Gamma$ and $r_{c}$ values.

\section{CONCLUSIONS}

Using the GB variational method with the OCP reference system, we obtained the plasma parameter, $\Gamma$, which gives the minimum of the Helmholtz free energy for liquid $3 \mathrm{~d}$ transition metals. This value of $\Gamma$ is about
105 for all the metals presently studied. Thus it turns out that the validity of the empirical value of $\Gamma=110$, with which the previous works /10-12/ were done, is well supported by the GB method. Then effective valencies, $Z_{s}$, were estimated from the relation of $Z_{s}=\sqrt{\Gamma a k_{\mathrm{B}}} T$ with $\Gamma=105$. With these $Z_{s}, \Gamma$ and $r_{c}$, the longwavelength limits of the structure factor, $s(0)$, are calculated using the random phase approximation. Agreement between theoretical and experimental $s(0)$ is quite good. We believe that this is firm evidence for the usability of the OCP model.

However, the present approach does not include the direct contributions of the d-electrons, although the relative number of the s- and d-electrons is taken into account. Even if leaving out the role of the bonding by the d-electrons, their effect is well absorbed in other terms, and the final results indicate that such a treatment is at least adequate for the structure-dependent properties. Therefore, when the pseudopotential parameters are properly selected, the simple-metal theory coupled with the OCP model appears to make some substantial contribution to our understanding of the thermodynamic properties of liquid transition metals.

\section{ACKNOWLEDGEMENT}

The authors are grateful to Professor Y. Waseda for a fruitful discussion on this work.

\section{REFERENCES}

1. Hausleitner, C. and Hafner, J., J. Phys., F18, 1025 (1988).

2. Hausleitner, C., Kahl, G. and Hafner, J., J. Phys. Condens. Matter, 3, 1589 (1991).

3. Bretonnet, J.L., Bhuiyan, G.M. and Silbert, M., J. Phys. Condens. Matter, 4, 5359 (1992).

4. Bretonnet, J.L. and Silbert, M., Phys. Chem. Liq., 24, 169 (1992).

5. Regnaut, C., Z. Phys. B-Condensed Matter, 76, 179 (1989).

6. Chaturvedi, D.K., Senatore, G. and Tosi, M.P., 
Lett. Nuovo Cimento, 30, 47 (1981).

7. Chaturvedi, D.K., Senatore, G. and Tosi, M.P., Nuovo Cimento, B62, 375 (1981).

8. Chaturvedi, D.K., Rovere, M., Senatore, G. and Tosi, M.P., Physica, B111, 11 (1981).

9. Itami, T. and Shimoji, M., J. Phys., F14, L15 (1984).

10. Itoh, H., Yokoyama, I. and Waseda, Y., J. Phys., F16, LI 13 (1986).

11. Yokoyama, I. and Naito, S., Physica, B 154, 309 (1989).

12. Yokoyama, I. and Naito, S., Physica, B 168, 229 (1991).

13. Wills, J.M. and Harrison, W.A., Phys. Rev., B28, 4363 (1983).

14. Hasegawa, M. and Watabe, M., J. Phys. Soc. Japan, 32, 14 (1972).

15. Jones, H., J. Chem. Phys., 55, 2640 (1971).
16. Jones, H., Phys. Rev., A8, 3215 (1973).

17. Young, W.H., J. Phys., F12, L19 (1982).

18. Ashcroft, N.W. and Stroud, D., Solid State Phys., 33 (New York: Academic) (1978).

19. Hasegawa, M. and Young, W.H., J. Phys., F11, 977 (1981).

20. Ashcroft, N.W., Phys. Lett., 23, 48 (1966).

21. Geldart, D.J.W. and Vosko, S.H., Can. J. Phys., 44, 2137 (1966).

22. Harrison, W.A., Electronic Structure and the Properties of Solids: The Physics of the Chemical Bond (San Francisco: Freeman) (1980).

23. Waseda, Y. and Ueno, S., Sci. Rep. Res. Inst. Tohoku Univ., 34A, 1 (1987).

24. Gray, P., Yokoyama, I. and Young, W.H., J. Phys., F10, 197 (1980).

25. Moruzzi, V.L., PhD Thesis, Technische Universität Wien (1985). 